\title{
Neurotransmitter Detection Using Corona Phase Molecular Recognition on Fluorescent Single-Walled Carbon Nanotube Sensors
}

\author{
Sebastian Kruss, ${ }^{\dagger}$ Markita P. Landry, ${ }^{\dagger}$ Emma Vander Ende, Barbara M.A. Lima, Nigel F. Reuel, \\ Jingqing Zhang, Justin Nelson, Bin $\mathrm{Mu}$, Andrew Hilmer, and Michael Strano* \\ Department of Chemical Engineering, Massachusetts Institute of Technology, Cambridge, Massachusetts 02139, United States
}

\section{Supporting Information}

ABSTRACT: Temporal and spatial changes in neurotransmitter concentrations are central to information processing in neural networks. Therefore, biosensors for neurotransmitters are essential tools for neuroscience. In this work, we applied a new technique, corona phase molecular recognition (CoPhMoRe), to identify adsorbed polymer phases on fluorescent single-walled carbon nanotubes (SWCNTs) that allow for the selective detection of specific neurotransmitters, including dopamine. We functionalized and

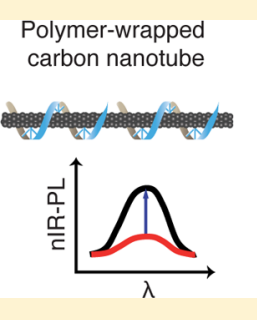

Single carbon

nanotube fluorescent sensor suspended SWCNTs with a library of different polymers $(n=30)$ containing phospholipids, nucleic acids, and amphiphilic polymers to study how neurotransmitters modulate the resulting band gap, near-infrared (nIR) fluorescence of the SWCNT. We identified several corona phases that enable the selective detection of neurotransmitters. Catecholamines such as dopamine increased the fluorescence of specific single-stranded DNA- and RNAwrapped SWCNTs by $58-80 \%$ upon addition of $100 \mu \mathrm{M}$ dopamine depending on the SWCNT chirality $(n, m)$. In solution, the limit of detection was $11 \mathrm{nM}\left[K_{\mathrm{d}}=433 \mathrm{nM}\right.$ for $(\mathrm{GT})_{15}$ DNA-wrapped SWCNTs]. Mechanistic studies revealed that this turn-on response is due to an increase in fluorescence quantum yield and not covalent modification of the SWCNT or scavenging of reactive oxygen species. When immobilized on a surface, the fluorescence intensity of a single DNA- or RNA-wrapped SWCNT is enhanced by a factor of up to $5.39 \pm 1.44$, whereby fluorescence signals are reversible. Our findings indicate that certain DNA/ RNA coronae act as conformational switches on SWCNTs, which reversibly modulate the SWCNT fluorescence. These findings suggest that our polymer-SWCNT constructs can act as fluorescent neurotransmitter sensors in the tissue-compatible nIR optical window, which may find applications in neuroscience.

\section{INTRODUCTION}

Neurotransmitter release is the basis of neurotransmission in chemical synapses and a central part of data processing in the brain. Therefore, the spatiotemporal concentration profile of neurotransmitters is of intrinsic interest for neuroscience and necessary to fully understand neural networks. Many diseases are directly related to altered patterns of neurotransmission, including Parkinson's disease, which is directly linked to depletion of dopaminergic neurons and low levels of the neurotransmitter dopamine. ${ }^{1}$ However, few analytical methods exist that can measure neurotransmitter gradients with high spatial and temporal resolution. In this work, we employed corona phase molecular recognition (CoPhMoRe) to develop novel fluorescent sensors for neurotransmitters. This technique involves screening and selection of a library of nanoparticleadsorbed organic phases, or coronae, for their ability to distinguish specific molecules. $^{2}$

Semiconducting single-walled carbon nanotubes (SWCNTs) conjugated with polymers are a new class of biosensors. ${ }^{3}$ SWCNTs consist only of a carbon monolayer. Therefore, small perturbations in the nanotube environment, which we call the nanotube corona, can be used to detect single-molecule analytes. ${ }^{4}$ SWCNTs fluoresce in the near-infrared (nIR), which is optimal for biomedical applications because it falls into the optical tissue transparency window. ${ }^{5}$ SWCNTs can be dispersed in various polymers, and this non-covalent functionalization renders the sensor biocompatible, water-soluble, and specific for certain analytes. Recently, sensors for reactive oxygen species (ROS), nitric oxide (NO), glucose, and DNA have been developed. ${ }^{4,6-9}$

The detection of neurotransmitters is very challenging for various reasons: Interfering substances such as chemical or structural neurotransmitter analogues prevent sensitive detection, whereas the short time scales, small length scales, and low neurotransmitter concentrations make it difficult to observe neurotransmitter release in real time. ${ }^{10-12}$ Nevertheless, sensors are necessary tools to study neurotransmitter release on the single-cell/synapse level and also in small networks of neurons or in vivo.

Electrochemical methods such as amperometry or cyclic voltammetry have provided valuable information on redoxactive neurotransmitters such as dopamine, epinephrine, and serotonin. Many biological studies were only possible because these methods provided quantitative information about neurotransmitter concentrations around cells, in brain slices, and in

Received: October 10, 2013

Published: December 19, 2013 
vivo. ${ }^{10,13}$ However, electrochemical methods are limited to molecules that can be oxidized or reduced at the electrode. Moreover, electrodes are usually large compared to the site of neurotransmitter release, so parallel/spatial detection of neurotransmitter release has not been achieved at the scale of the synapse. Other approaches for neurotransmitter detection are based on fluorescent labeling. Neurotransmitter-containing vesicles can be labeled with dyes, and the fusion of these vesicles with the synaptic cell membrane can be observed by fluorescence microscopy. Vesicles that contain neurotransmitters have different $\mathrm{pH}$ values compared with the extracellular space. As such, $\mathrm{pH}$-dependent fluorescent proteins/dyes have been used to visualize exocytosis. ${ }^{11}$ However, this approach lacks direct optical neurotransmitter identification and rather visualizes structural changes of vesicles and membranes instead of directly visualizing the neurotransmitter itself. In contrast, fluorescent false neurotransmitters (FFNs) are fluorescent dyes having chemical structures that are similar to the neurotransmitter of interest and are loaded into synaptic vesicles by neurotransmitter transporters. This method was used to visualize fluorescence depletion upon stimulation in single synapses of dopaminergic neurons ${ }^{14,15}$ but does not directly detect natural neurotransmitters. Another approach is based on modifying biological recognition units of neurotransmitters and conjugating them with fluorescent dyes. This method was used to engineer $\gamma$-aminobenzoic acid (GABA) sensors and glutamate sensors. ${ }^{16,17}$ Recently, glutamate was measured by using green fluorescent protein (GFP)-conjugated glutamate receptors that can be also transfected into cells. ${ }^{18}$ While these efforts have provided us with many tools to study neuronal pathways, there is still a great need for a direct high-resolution spatiotemporal detection tool for neurotransmitters, which will enable completely new insights into how neurons communicate with each other.

Here we present a new fluorescent sensor platform for the optical detection of neurotransmitters that is based on the nIR fluorescence of SWCNTs. Fluorescent sensors with distinct sensitivity and specificity for different neurotransmitters were synthesized by wrapping carbon nanotubes with different polymers. The specificity of these sensors does not arise from using known biological recognition elements. Rather, it is based on the unique conformational changes that happen when a polymer is pinned at a nanoparticle surface, creating a defined corona. Only the combination of a polymer and its confinement on the nanoparticle cause recognition and signal transduction in the presence of the analyte according to this scheme, which we have introduced previously as corona phase molecular recognition (CoPhMoRe). ${ }^{2}$

We show that catecholamines such as dopamine and epinephrine increase the fluorescence of DNA- or RNAwrapped carbon nanotubes by enhancing their fluorescence quantum yield. This fluorescence increase is an important finding with respect to carbon nanotube photophysics, as it presents the first example of CoPhMoRe that leads to a fluorescence turn-on signal. We further show how these underlying mechanisms are the founding principle for a fluorescent turn-on sensor for dopamine.

\section{EXPERIMENTAL SECTION}

Materials. Chemicals, including the neurotransmitters, were purchased from Sigma-Aldrich (USA), unless stated otherwise. Single-stranded DNA (ssDNA) and RNA (ssRNA) sequences (N1N13) were purchased from IDT (USA). HiPCO SWCNTs were purchased from Unidym and processed according to the suggestions by the manufacturer (extraction of non-SWCNT material by phase separation in water/hexane). Phospholipids were purchased from Avanti Polar Lipids (USA). Compounds P1-P4 were synthesized as previously reported. ${ }^{2,19}$ Compound P5 was purchased from Polyscitech (USA).

Preparation and Characterization of Wrapped SWCNTs. We used direct probe-tip sonication (Cole Parmer) to encapsulate HiPCO SWCNTs (Unidym) with the several nucleic acid derivatives used in this work (10 min, $40 \%$ amplitude). For this purpose, the nucleic acid was dissolved in a $0.1 \mathrm{M} \mathrm{NaCl}$ solution $(100 \mathrm{mg} / \mathrm{mL})$. Next, $20 \mu \mathrm{L}$ of the DNA solution was added to $980 \mu \mathrm{L}$ of $0.1 \mathrm{M} \mathrm{NaCl}$ solution and 1 mg HiPCO SWCNTs, and the resulting suspension was tip-sonicated for $10 \mathrm{~min}$ (3 mm tip diameter, 40\% amplitude) in an ice bath. After sonication, we centrifuged the samples two times for $90 \mathrm{~min}$ at $16100 \mathrm{~g}$ and collected the supernatant. For all other polymers we used dialysis: First, sodium cholate (SC)-wrapped SWCNTs were prepared by tip sonication of $2 \mathrm{wt} \% \mathrm{SC}$ in water $(6 \mathrm{~mm}$ tip diameter, $40 \%$ amplitude, $1 \mathrm{~h}$ ). These samples were centrifuged at $150000 \mathrm{~g}$ (Beckmann Coulter ultracentrifuge) for $4 \mathrm{~h}$. Second, the polymer (1 wt \%) was dissolved in a solution of SC-wrapped SWCNTs and dialyzed for 5 days against water in a $3.5 \mathrm{kDa}$ molecular-weight cutoff dialysis bag (Spectra/Por dialysis membrane). All of the samples were analyzed by UV-vis-nIR spectroscopy (Shimadzu UV-3101PC).

CoPhMoRe Screening and Solution-Based Experiments. Polymer-wrapped SWCNTs were diluted in phosphate-buffered saline (PBS) (pH 7.4, $10 \mathrm{mM}$ ) to a SWCNT concentration corresponding to an absorbance of 0.036 at $632 \mathrm{~nm}$. To 96 well-plates (Microtest 96 tissue culture plate, $\mathrm{BD}$ ) were added $198 \mu \mathrm{L}$ aliquots of this solution. The nIR fluorescence was collected on a Zeiss AxioVision inverted microscope coupled to a PI Acton SP2500 spectrometer and a Princeton Instruments InGaAs OMA V array detector. The sample was excited with a $785 \mathrm{~nm}$ photodiode laser $(450 \mathrm{~mW})$. Exposure times were $10 \mathrm{~s}$. The neurotransmitter was dissolved in PBS $(10 \mathrm{mM}$ stock solution), and $2 \mu \mathrm{L}$ of the solution was added into the wells at a final concentration of $100 \mu \mathrm{M}$ or smaller. In a typical experiment, the fluorescence responses of nine different neurotransmitters for one polymer-SWCNT combination were measured in triplicate $(n=3)$. The dopamine solution was freshly prepared and used within $10 \mathrm{~min}$. Subsequent measurement of the samples in the well plate took around $10 \mathrm{~min}$. The experiments with ROS scavengers were done similarly: Spectra were collected before addition of the scavengers, after addition of the scavengers, and after addition of dopamine $(100 \mu \mathrm{M})$. All of the experiments were performed in PBS. We added neurotransmitters and other compounds in small volumes ( 1 vol \%), which changed the SWCNT concentration only slightly $(\sim 1 \%)$.

Microscopy Setup. Visible-Wavelength TIRF Microscopy. The total internal reflection fluorescence (TIRF) microscope uses a supercontinuum excitation source (NKT, SuperK Extreme EXR15), which is relayed through several conditioning optics, including a $10 \times$ beam expansion telescope to expand the diameter of the excitation beam to approximately $1.5 \mathrm{~cm}$. This allows overfill into the back aperture of the microscope objective and enables maximal excitation area of the sample. The expanded beam is directed into a plano-convex focusing lens (TIR lens), which is mounted on a three-axis translational stage [Figure S3a in the Supporting Information (SI)]. The angle of beam incidence $(\Theta)$ is changed by moving the TIR lens in the plane perpendicular to beam propagation. When $\Theta=\Theta_{c}$ (the critical angle), all of the incident light is reflected from the surface of the glass-water interface and generates an evanescent field that excites fluorophores in the vicinity of the glass-water interface.

Before light enters the microscope objective, a $531 \mathrm{~nm}$ narrowbandpass filter (FF01-531/40-25, Semrock) tunes the excitation wavelength relayed to the sample through the objective. A $562 \mathrm{~nm}$ dichroic mirror (FF562-Di03-25 $\times$ 36, Semrock) reflects this excitation wavelength while passing the $\mathrm{Cy} 3$ characteristic emission by passing wavelengths above $\sim 562 \mathrm{~nm}$. The emission path of the prism-TIRF microscope begins with the collection of the emission from the sample by the objective. This emission passes through a 593 $\mathrm{nm}$ bandpass filter (FF01-593/40-25, Semrock) that reflects the 
a)
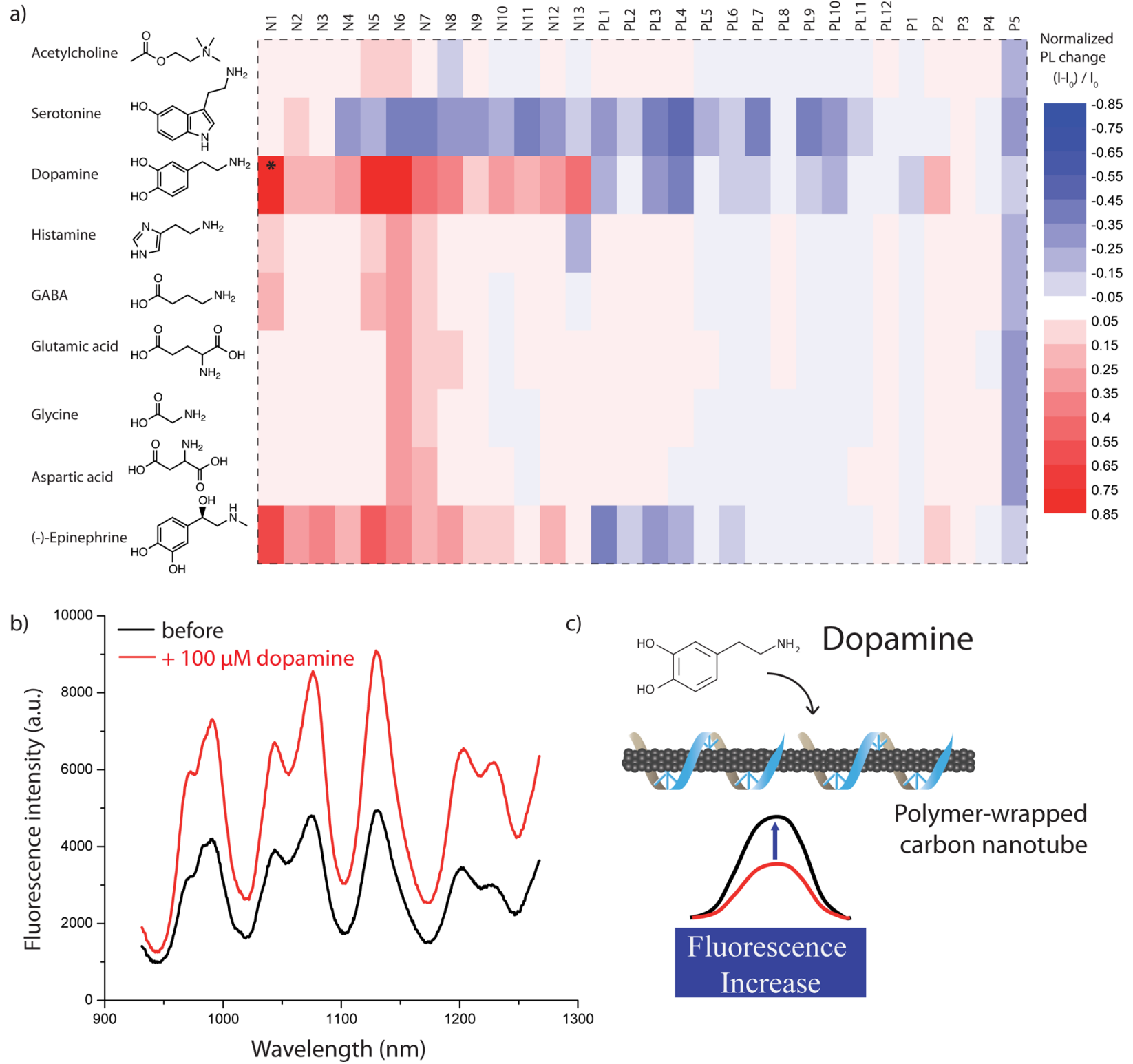

Polymer-wrapped carbon nanotube

Figure 1. Screening of SWCNT-polymer conjugates for fluorescence modulation by neurotransmitters. (a) Fluorescence responses of polymerSWCNT conjugates $\left(x\right.$ axis) to neurotransmitters $\left(y\right.$ axis). The normalized fluorescence changes $\left(I-I_{0}\right) / I_{0}$ of different polymer-SWCNT conjugates upon addition of different neurotransmitters $(100 \mu \mathrm{M})$ are shown in a color-coded heat map. Polymer structures are shown in Figure 2. N1-N13 are nucleic acids, PL1-PL12 are phospholipids, and P1-P5 are amphiphilic polymers. Red indicates a fluorescence increase and blue a fluorescence decrease. (b) nIR fluorescence spectrum (785 nm excitation) of (GT) ${ }_{15}-$ SWCNT before and after addition of $100 \mu \mathrm{M}$ dopamine in PBS. The fluorescence intensity increased by up to $80 \%$ depending on the chirality of the SWCNTs. The distinct peaks correspond to different SWCNT chiralities in the sample. (c) Schematic of the fluorescent turn-on sensor for dopamine.

excitation laser light $(532 \mathrm{~nm})$ but transmits the fluorescence emission wavelength of the $\mathrm{Cy} 3$ dye $(570 \mathrm{~nm})$. A pair of doublet lenses expand the $75 \mu \mathrm{m} \times 75 \mu \mathrm{m}$ image to fill the CCD sensor $(8.2 \mathrm{~mm} \times 8.2 \mathrm{~mm}$; iXon3 EMCCD, Andor Technology).

nIR Epifluoresence Microscopy. Single-SWCNT data were collected on an inverted microscope (Zeiss Axiovert 200) equipped with a $100 \times$ objective (Zeiss, $\alpha$-Plan-APOCHROMAT $100 \times / 1.46$ Oil DIC (UV) VIS-IR) attached to a 2D InGaAs CCD array (OMA-V 2D, Princeton Instruments) (SI Figure S3b). Fluorescence was excited by a $658 \mathrm{~nm} 200 \mathrm{~mW}$ diode-pumped solid-state laser (CrystaLaser, RCL100-660).

Microfluidic Chamber Preparation. Microfluidic chambers for visible TIRF experiments were surface-passivated with bovine serum albumin (BSA) to avoid nonspecific adsorption of the fluorescently tagged biomolecules to the glass surface, as previously described. ${ }^{20}$ The formation of microfluidic flow channels was achieved by cutting several channels out of double-sided tape and sealing the tape between the quartz slide and the coverslip.

For flow experiments, a micropipet tip was introduced at the flow inlet of a single microfluidic channel, and a syringe was coupled into the flow outlet to change the buffer conditions of the experimental chamber. To passivate these chambers, BSA coating of the glass slide surface and a glass coverslip was performed by flowing in a $100 \mathrm{nM}$ solution of BSA tagged with biotin (BSA-Bt). ${ }^{21}$ Subsequently, PBS buffer was flowed through the microfluidic channel to rinse nonadhered BSA-Bt. Next, a $33 \mathrm{nM}$ solution of neutravidin protein was flowed in and allowed to incubate for $10 \mathrm{~min}$ for binding to the biotin moiety on the surface-bound BSA-Bt. Neutravidin protein bound nonspecifically to the SWCNT surface, allowing surface immobilization of the DNA-wrapped SWCNT samples within the microfluidic chambers. 


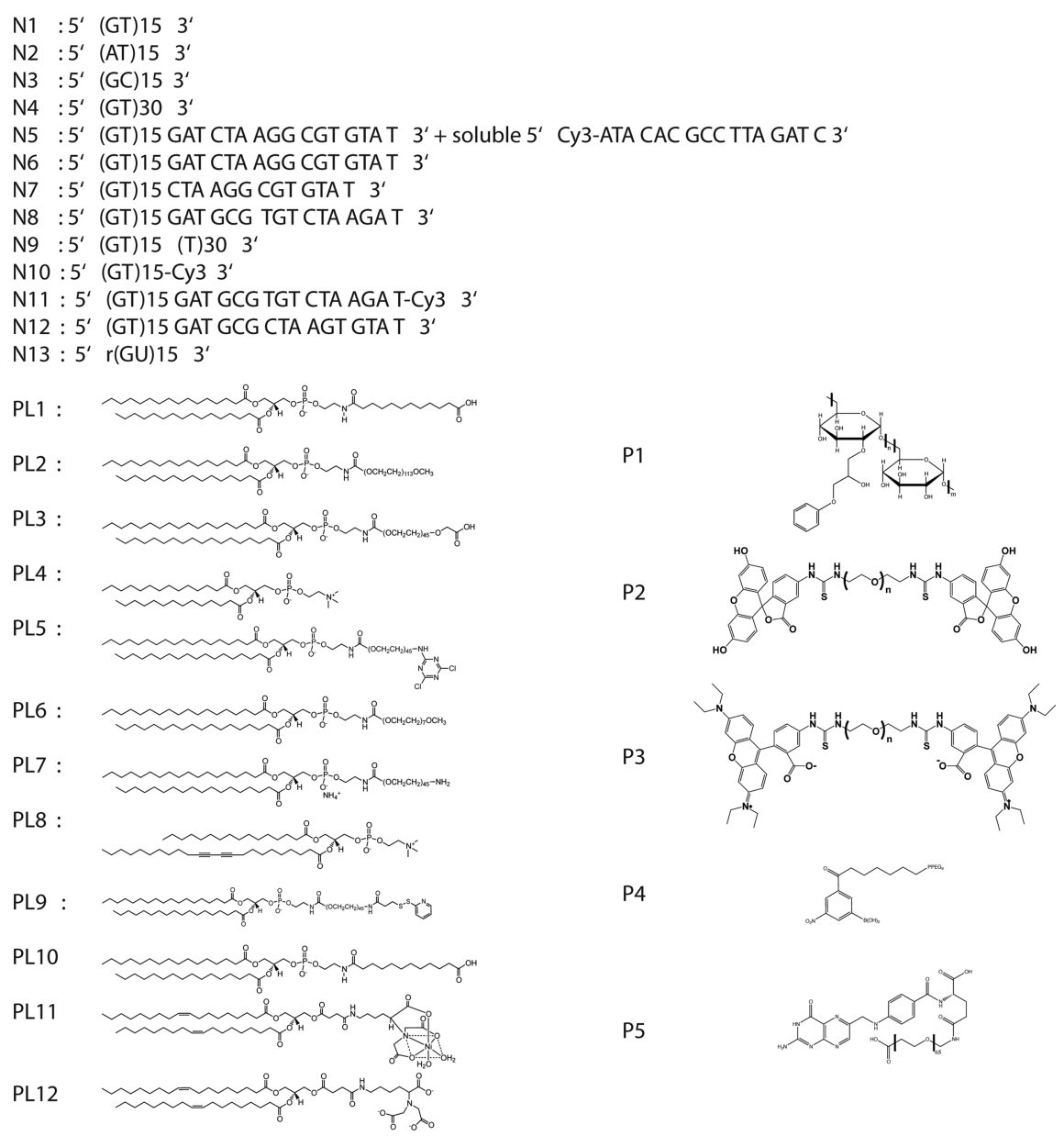

Figure 2. Polymers that were used to suspend/functionalize SWCNTs and generate a sensor library. N1-N12 are ssDNA sequences, N13 is an ssRNA sequence, PL1-PL12 are phospholipids with different head and tail groups, and P1-P5 are amphiphilic polymers with hydrophobic and hydrophilic groups.

For reversibility experiments, ibidi flow chambers were used (ibidi, sticky-Slide VI 0.4) and attached to a pump (Ismatec). Glass substrates were functionalized with (3-aminopropyl)triethoxysilane (APTES) in ethanol (1\% APTES, $1 \%$ water) and then mounted under the flow chamber. Next, $1 \mathrm{mg} / \mathrm{L}(\mathrm{GT})_{15}$ or $(\mathrm{GU})_{15}$-wrapped SWCNT solution in PBS was incubated in the channels to adsorb SWCNTs to the surface. For the reversibility and single-SWCNT experiments, dopamine $(100 \mu \mathrm{M})$ and PBS were periodically washed over the surface (flow rate $50 \mu \mathrm{L} / \mathrm{min}$ ).

\section{RESULTS}

CoPhMoRe Screening for Polymer-SWCNT Conjugates That Change Fluorescence upon Neurotransmitter Addition. We suspended HiPCO SWCNTs in various polymers (see the Experimental Section) to solubilize them in aqueous solution, render them sensitive for neurotransmitters, and measure the influence of neurotransmitters on their fluorescence spectra (Figure 1). We elected to use unseparated SWCNTs in order to detect band gap- or diameter-dependent analyte responses. For this purpose, we used either direct ultrasonication or dialysis of SC-wrapped carbon nanotubes. The structures are shown in Figure 2. The polymers are abbreviated as follows: N1-N12 are ssDNA sequences, N13 is the ssRNA sequence $(\mathrm{GU})_{15}$, and PL1-PL12 are phospholipids with different head and tail groups. P1-P5 are different amphiphilic polymers with a hydrophobic group that binds to the carbon surface and a hydrophilic group that renders the conjugate water-soluble. All of the SWCNT-polymer hybrid combinations were characterized by absorption spectroscopy. Samples were then diluted to a final concentration of $1 \mathrm{mg} / \mathrm{L}$ in $\mathrm{PBS}$ at $\mathrm{pH}$ 7.4. SWCNT-polymer conjugates were excited by a $785 \mathrm{~nm}$ photodiode laser, and their emission spectra between 950 and $1250 \mathrm{~nm}$ were recorded. Fluorescence spectra were collected before and after addition of the neurotransmitters (final concentration $100 \mu \mathrm{M}$ ). The library of neurotransmitters included acetylcholine, serotonin, dopamine, histamine, GABA, glutamic acid, glycine, aspartic acid, and (-)-epinephrine.

Fluorescence changes were calculated by comparing the SWCNT intensities at the fluorescence emission peak of the $(9,4)$ chirality SWCNTs (around $1132 \mathrm{~nm}$ for nucleic acidwrapped SWCNTs) because in most cases the $(9,4)$ peak provided the largest fluorescence count in our setup. The sensor response was defined as $\left(I-I_{0}\right) / I_{0}$, that is, the difference between the final fluorescence intensity $I$ and the starting intensity $I_{0},\left(I-I_{0}\right)$, normalized by the starting intensity $I_{0}$. For all of the polymers (Figure 2), fluorescence intensity modulations but no wavelength peak shifts were observed. The optical responses for the different SWCNTpolymer hybrids in the presence of various neurotransmitters are shown in Figure 1. The fluorescence changes are colorcoded $\left(\Delta I / I_{0}>0\right.$, red; $\Delta I / I_{0}<0$, blue $)$, and the intensity of the color corresponds to the absolute intensity of the response. We found several SWCNT-polymer conjugates with clear 

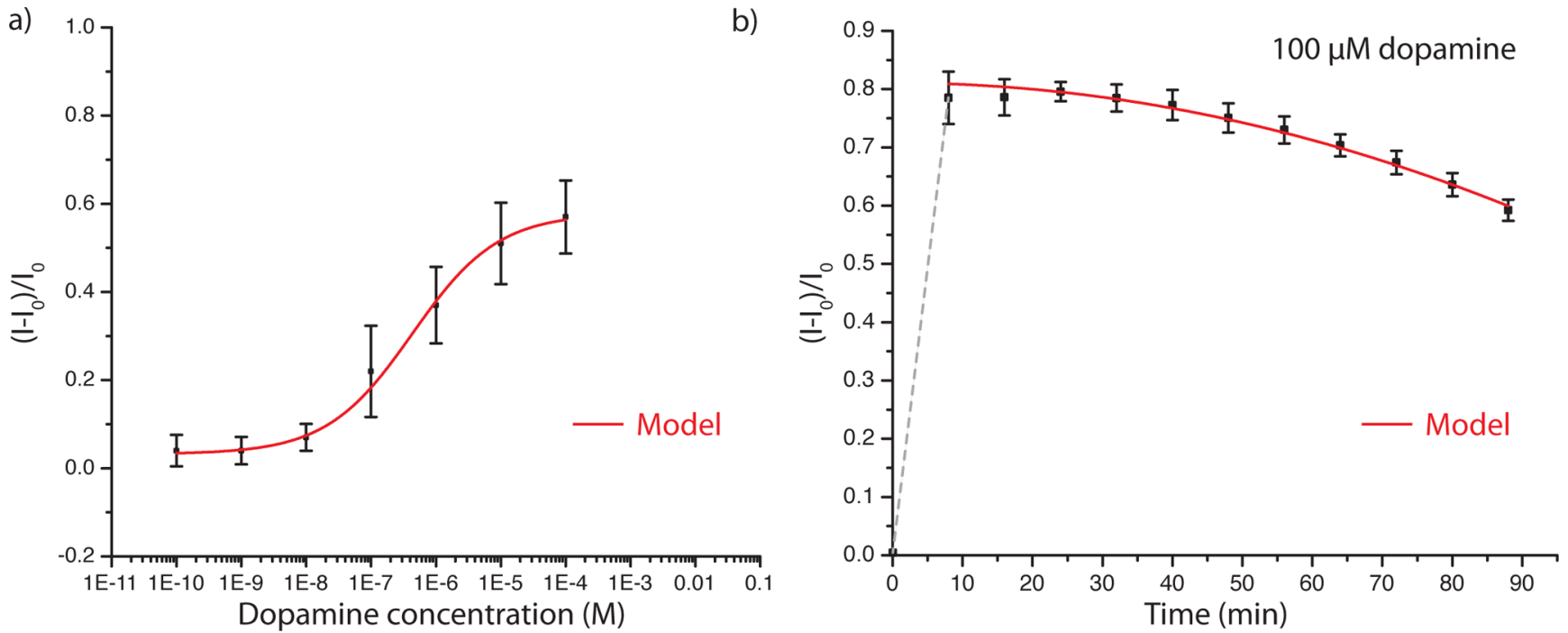

Figure 3. Calibration curve and dopamine response kinetics of the $(\mathrm{GT})_{15}-\mathrm{SWCNT}$ sensor. (a) Calibration curve of the dopamine sensor in solution (PBS), $n=3$, three replicates each. (b) Time-dependent response after addition of $100 \mu \mathrm{M}$ dopamine (at $t=0$ min, the starting intensity was measured). The decrease of fluorescence is most probably related to dopamine polymerization. Errors are standard deviations.

modulations in nIR fluorescence upon neurotransmitter addition.

All of the DNA- or RNA-wrapped SWCNTs (N1-N13) showed an increase in fluorescence from $11 \%$ to $80 \%$ upon addition of dopamine and $3 \%$ to $62 \%$ for (-)-epinephrine. Most nucleic acid-wrapped SWCNTs showed a fluorescence decrease upon addition of $100 \mu \mathrm{M}$ serotonin. In general, aromatic (and redox-active) neurotransmitters such as dopamine showed stronger responses than non-redox-active neurotransmitters such as glycine. The SWCNT-polymer conjugate with the strongest fluorescence increase, N1, corresponds to $(\mathrm{GT})_{15}$ DNA-wrapped SWCNTs. We used this specific DNA sequence as an exemplary model to study the fluorescence enhancement of nucleic acid-SWCNT conjugates to dopamine and to investigate the mechanism of this molecular recognition. In particular, we focused on dopamine because it is one of the central neurotransmitters in the brain and is linked to reward processing and learning. ${ }^{22}$

In Figure 1b, the fluorescence spectrum of $(\mathrm{GT})_{15}-\mathrm{SWCNT}$ in PBS before and after the addition of $100 \mu \mathrm{M}$ dopamine is shown. Upon addition of dopamine, the fluorescence increased by a factor of $58-81 \%$. Different peaks correspond to different chiralities of SWCNTs that are present in a typical HiPCO sample, where the fluorescence response depended on the chirality $(n, m)$ of the SWCNTs $[(6,5), 991 \mathrm{~nm}, 0.80 \pm 0.12$; $(7,5), 1044 \mathrm{~nm}, 0.63 \pm 0.088$; $(10,2), 1077 \mathrm{~nm}, 0.66 \pm 0.08$; $(9,4), 1132 \mathrm{~nm}, 0.73 \pm 0.11 ;(8,6), 1203 \mathrm{~nm}, 0.58 \pm 0.09]$. Dopamine changed the SWCNT fluorescence intensity but did not shift the spectrum. Figure 1c shows the basic principle of this sensor platform: a polymer is pinned to a carbon nanotube surface and mediates the interaction of the analyte with the SWCNT corona.

To determine the source of increased SWCNT fluorescence upon dopamine addition, we took absorption spectra of $(\mathrm{GT})_{15}$-SWCNT. The SWCNT absorption spectra did not change upon addition of $100 \mu \mathrm{M}$ dopamine (SI Figure S1a). Both the intensities and positions of the $E_{11}$ and $E_{22}$ optical transitions remained identical before and after dopamine addition. Therefore, the change in SWCNT fluorescence (Figure 1b) could not be attributed to changes in the absorption cross section upon dopamine addition and must be due to an increase in quantum yield of $(\mathrm{GT})_{15}-\mathrm{SWCNT}$ fluorescence.

Another potential source of SWCNT fluorescence change could be covalent modification of the SWCNT surface by dopamine. Dopamine is a reactive molecule that is known to polymerize readily in solution and could covalently modify the carbon surface and thus induce a change in SWCNT fluorescence. ${ }^{23,24}$ The Raman spectrum of a $1 \mathrm{mg} / \mathrm{L}(\mathrm{GT})_{15}-$ SWCNT sample in PBS before and $10 \mathrm{~min}$ after the addition of dopamine showed no difference in Raman intensity (SI Figure $\mathrm{S} 1 \mathrm{~b})$. The $\mathrm{G}$ peak at $1591 \mathrm{~cm}^{-1}$ and the $\mathrm{D}$ peak at $1307 \mathrm{~cm}^{-1}$ did not change upon dopamine addition. This invariance of the $\mathrm{G} / \mathrm{D}$ ratio indicates that no $\mathrm{sp}^{3}$ defects were introduced into the carbon lattice by dopamine.

Sensitivity and Kinetics of the Fluorescent SWCNTBased Dopamine Sensors. We used the peak of the $(9,4)$ SWCNTs to calculate the sensor response $\left(I-I_{0}\right) / I_{0}$ and determine a calibration curve (Figure $3 \mathrm{a}$ ). Even at dopamine concentrations of $100 \mathrm{pM}$, a fluorescence increase of $5 \%$ was observed. The approximate linear regime of the sensor was between $10 \mathrm{nM}$ and $10 \mu \mathrm{M}$, on the basis of the sensor response at $10 \mu \mathrm{M}, 1 \mu \mathrm{M}, 100 \mathrm{nM}$, and $10 \mathrm{nM}$ (linear fit on a logarithmic scale, $\left.R^{2}>0.98\right)$. At higher concentrations $(>10$ $\mu \mathrm{M})$, the sensor response saturated. The calibration curve can be described by a kinetic adsorption model in which $\mathrm{A}$ is the analyte (dopamine) and $\theta$ is an available recognition site on the SWCNT sensor. The relationship between the analyte and available docking sites for dopamine can be described as follows:

$$
\mathrm{A}+\theta \rightleftarrows \mathrm{A} \theta
$$

As we show in later single-molecule results, this turn-on response is instantaneous. Therefore, the equilibrium for this reaction can be modeled as an instantaneous reaction and can be described by the following equilibrium constant:

$$
K_{\mathrm{A}}=\frac{[\mathrm{A} \theta]}{[\mathrm{A}][\theta]}
$$


a)

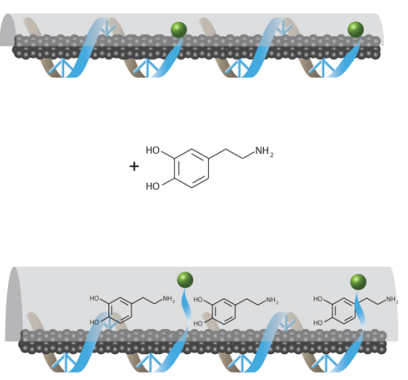

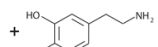
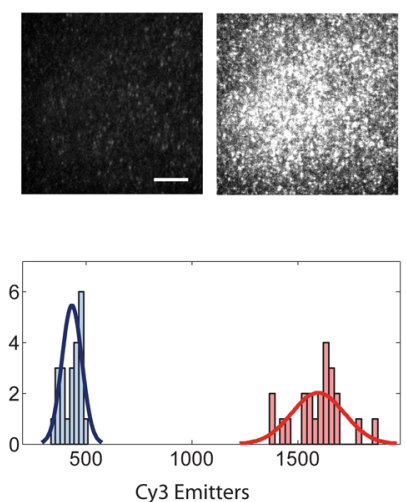

b)
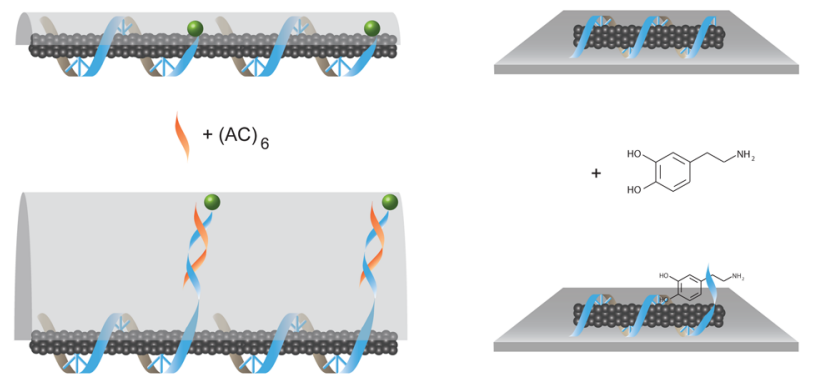

c)

d)
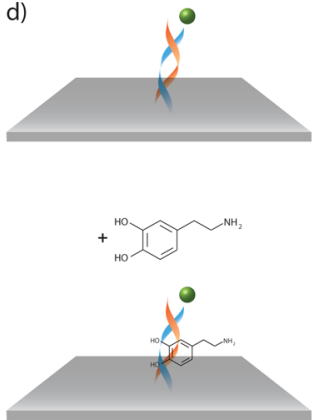
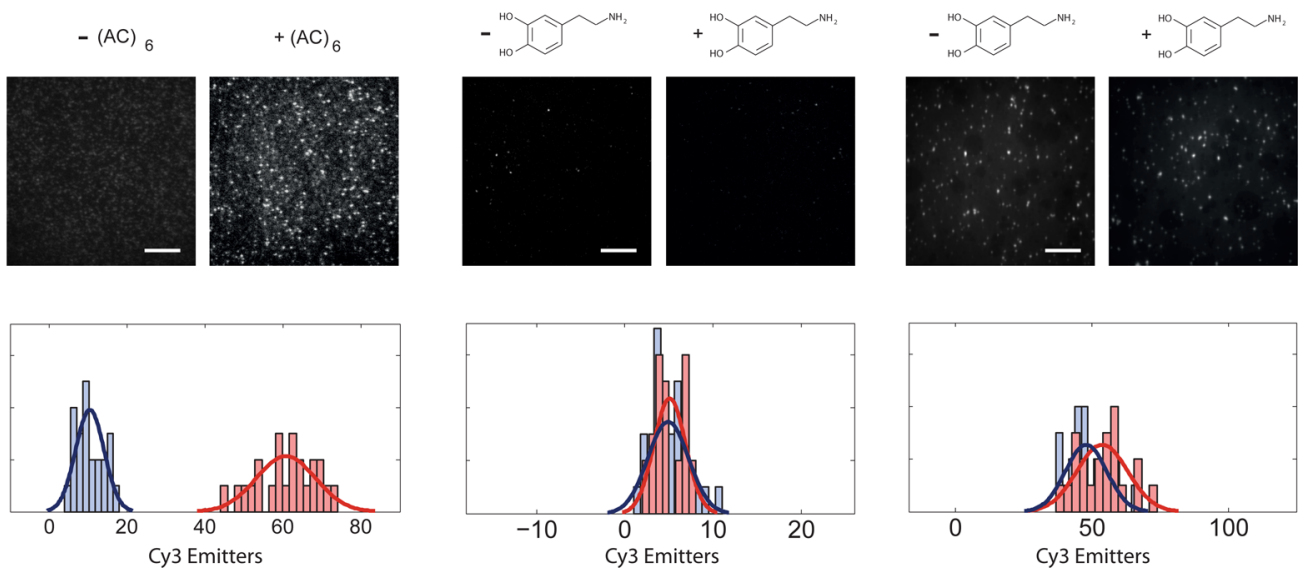

Figure 4. Dopamine-induced conformational shift of DNA. (GT) ${ }_{15}$ DNA was 5 ' terminally tagged with a Cy3 fluorophore, conjugated to a SWCNT, and immobilized on a surface prior to flowing in dopamine. (a) Upon addition of $100 \mu \mathrm{M}$ dopamine, dequenching of the Cy3 fluorophore due to fluorophore destacking was observed. (b) A positive control showed similar fluorophore dequenching when the short unlabeled complementary oligonucleotide $(\mathrm{AC})_{6}(50 \mathrm{nM})$ was added to the system and allowed to hybridize to the Cy3-labeled end of the DNA on the SWCNT. (c) A negative control with unlabeled DNA showed no signal before or after dopamine addition. (d) A negative control with Cy3-labeled DNA on a surface without the SWCNT also showed no change in Cy3 fluorescence upon addition of dopamine. The histograms show the fluorophore counts before (blue) and after (red) addition of dopamine/(AC) 6 .

The total concentration of available recognition sites, $[\theta]_{\text {tot }}$ is the sum of the concentrations of the free and occupied sites:

$$
[\theta]_{\mathrm{tot}}=[\mathrm{A} \theta]+[\theta]
$$

We can use eqs 2 and 3 to derive the following expression for $[\theta]_{\text {tot }}$ in terms of the dopamine concentration $[\mathrm{A}]$ :

$$
\begin{aligned}
{[\theta]_{\text {tot }} } & =[\mathrm{A} \theta]+[\theta] \\
& =[\mathrm{A} \theta]+\frac{[\mathrm{A} \theta]}{[\mathrm{A}] K_{\mathrm{A}}} \\
& =[\mathrm{A} \theta]\left(1+\frac{1}{[\mathrm{~A}] K_{\mathrm{A}}}\right) \\
& =[\mathrm{A} \theta]\left(\frac{[\mathrm{A}] K_{\mathrm{A}}+1}{[\mathrm{~A}] K_{\mathrm{A}}}\right)
\end{aligned}
$$

If we assume that the sensor response (i.e., the normalized intensity change) is proportional to the $\mathrm{A} \theta / \theta_{\text {tot }}$ ratio, we find that

$$
\frac{I-I_{0}}{I_{0}}=\alpha \frac{[\mathrm{A} \theta]}{\left[\theta_{\text {tot }}\right]}+B=\alpha \frac{\left([\mathrm{A}] K_{\mathrm{A}}\right)^{n}}{\left([\mathrm{~A}] K_{\mathrm{A}}\right)^{n}+1}+B
$$

where the parameter $B$ accounts for background and $n$ for cooperativity. Fitting the curve in Figure $3 a$ results in a proportionality factor $\alpha=0.55$ with $B=0.032, K_{\mathrm{d}}=1 / K_{\mathrm{A}}=433$ $\mathrm{nM}$, and $n=0.66$, indicating negative cooperativity $\left(R^{2}>0.99\right)$. The limit of detection was $11 \mathrm{nM}$; this value was calculated by adding the sensor response for the addition of only buffer (PBS) to 3 times the standard deviation (noise) and then using the fit to eq 5 to calculate a concentration (limit of detection) from this value.

We observed time-dependent variations in the absolute magnitude of the sensor response, which we attributed to the known phenomenon of dopamine polymerization in solution. $^{23,25,26}$ The time dependence of the sensor response can be explained in terms of a polymerization product $\mathrm{C}$ that quenches the SWCNT fluorescence. Dopamine (A) is oxidized to dopamine quinone $(\mathrm{B})$, which then polymerizes to give $\mathrm{C}$ :

$$
A \stackrel{k_{1}}{\rightarrow} \mathrm{B} \stackrel{k_{2}}{\rightarrow} \mathrm{C}
$$

Because of the time frame of the experiment, we can assume that $[\mathrm{A}] \gg[\mathrm{B}] \gg[\mathrm{C}]$ and neglect back-reactions. The first reaction does depend on oxidative species such as $\mathrm{O}_{2}$ and is therefore not dependent on $[\mathrm{A}]$. Therefore, we find that

$$
[\mathrm{B}]=k_{1} t
$$

$$
\frac{\mathrm{d}[\mathrm{C}]}{\mathrm{d} t}=k_{2}[\mathrm{~B}]
$$


a)

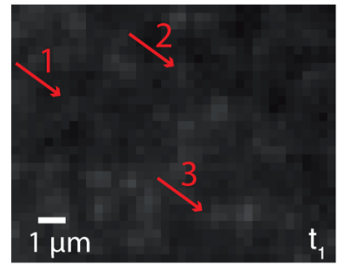

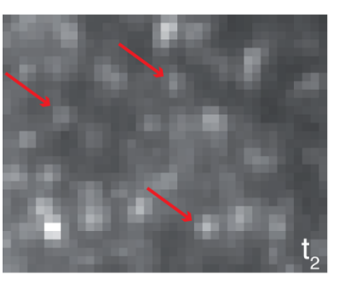

c)

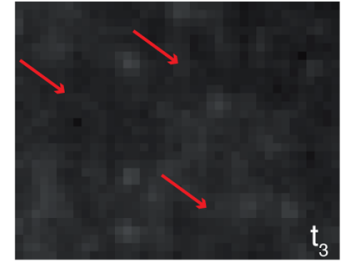

Sensor 2

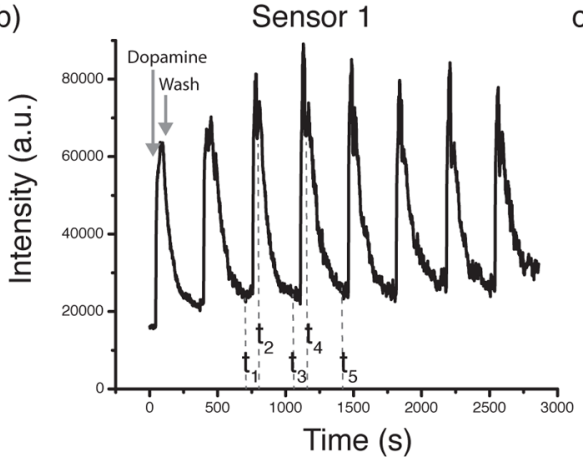

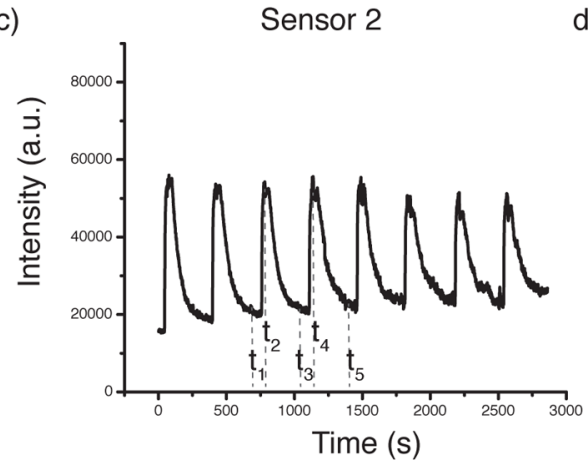

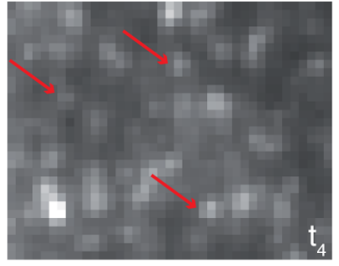

d)

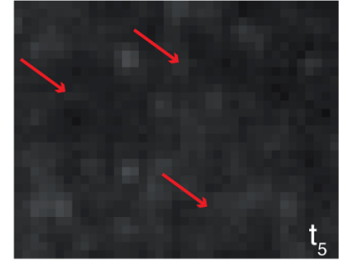

Sensor 3

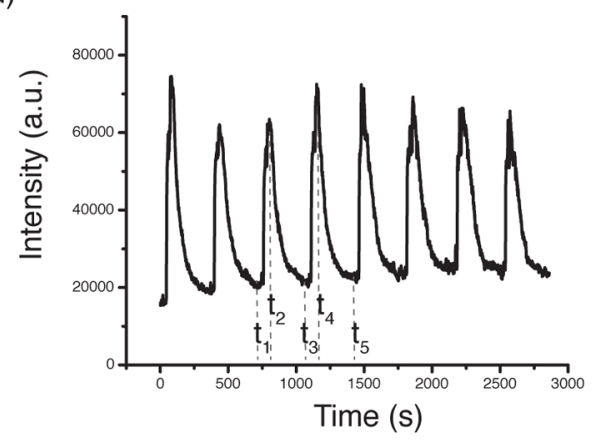

Figure 5. Reversible dopamine response of immobilized single $(\mathrm{GT})_{15}-\mathrm{SWCNT}$ sensors. (a) nIR fluorescence images of sensors that were immobilized to a surface in a flow chamber. The red arrows indicate three different carbon nanotubes, the intensities of which are shown in ( $b-d)$. The images are from the same spot at different time points, as indicated in $(\mathrm{b}-\mathrm{d})$. The surface was periodically exposed to $100 \mu \mathrm{M}$ dopamine and washed with PBS. $(\mathrm{b}-\mathrm{d})$ Intensity traces of 2 pixel $\times 2$ pixel $(585 \mathrm{~nm} \times 585 \mathrm{~nm})$ regions of the SWCNTs indicated by the red arrows in $(\mathrm{a})$. The sensor response was reversible with a small hysteresis. The sensor response is convoluted by the concentration profile of dopamine (mixing).

If we assume that the sensor response can be described by a linear combination of effects due to quenching (from $\mathrm{C}$ ) and dequenching (from A), we find that

$$
\left(\frac{I-I_{0}}{I_{0}}\right)_{t}=\left(\frac{I-I_{0}}{I_{0}}\right)_{t=0}+\beta t^{2}
$$

The parameter $\beta$ contains the rate constants and a quenching factor $(\beta<0)$. The sensor response over the time course of 90 min is shown in Figure $3 \mathrm{~b}$ [fit parameters: $\left(\left(I-I_{0}\right) / I_{0}\right)_{t=0}=$ 0.81 and $\left.\beta=-2.73 \times 10^{-5} \mathrm{~min}^{-2}\right)$. The initial fluorescence increase was always instantaneous. It was only limited by diffusion of dopamine to the sensor (see the single-SWCNT data below). However, the fluorescence response changed from $80 \%$ fluorescence increase to approximately $60 \%$ increase within 90 min of solubilizing dopamine in PBS buffer, which we attribute to the gradual polymerization of dopamine. ${ }^{23,25,26}$ Again, the absorption spectra of the SWCNTs were not affected by the dopamine polymerization within the first 90 min, suggesting that precipitation of the sensor or light absorption by polymerized dopamine did not play a role (SI Figure S2). Therefore, the age of the dopamine solution and the time needed to perform the measurements have an influence on the fluorescence change. Of note, a $10 \mathrm{mM}$ dopamine stock solution in PBS showed signs of polymerization, as evidenced by darkening of the solution within $1 \mathrm{~h}$. After 1 day, a black precipitate had formed. Therefore, we always prepared fresh dopamine solutions for all assays presented in this work and discarded dopamine solutions that were more than $10 \mathrm{~min}$ old (see the Experimental Section). Variations of the initial absolute response in independent experiments were most likely due to slight variations in the age of the dopamine solution and the time needed to measure all samples.

Conformational Changes of the Nucleic Acid Backbone during Dopamine Recognition. In the absence of covalent modification of the SWCNT by dopamine, and without ROS generation in the sensor system, we turned to single-molecule imaging of the sample to visualize any conformational changes that may accompany dopamine detection. We encapsulated the SWCNT sample with $5^{\prime}$ terminally labeled $(\mathrm{GT})_{15}$ DNA, where we used the dye Cy3 as a visible tag that reports on the structure of the $5^{\prime}$ end of the DNA. Quenching of organic fluorophores occurs as a function of proximity to the surface of the SWCNT, thereby creating a fluorescent ruler to quantify the degree of DNA desorption. ${ }^{27} \mathrm{~A}$ lack of $\mathrm{Cy} 3$ fluorescence is expected for a DNA polymer that remains adsorbed to the SWCNT surface (i.e., within a distance of $1 \mathrm{~nm}$ from the SWCNT surface). Conversely, bright Cy3 fluorescence is expected for DNA polymers that desorb from the SWCNT surface. We immobilized Cy3-labeled (GT) $)_{15}$ SWCNT complexes $\left[(\mathrm{GT})_{15}-\mathrm{Cy} 3-\mathrm{SWCNT}\right]$ to the surface of a microfluidic channel as described in the Experimental Section and employed proper passivation of the slide surfaces to avoid nonspecific interactions of molecules with the surface. To visualize the fluorescence signal from surface-adsorbed SWCNT sensors, we used a custom-built TIRF microscope. This excitation method drastically reduced the fluorescent background and enabled high-resolution observation of dynamic single-biomolecule behavior.

Immobilized $(\mathrm{GT})_{15}-\mathrm{Cy} 3-\mathrm{SWCNT}$ showed a highly quenched Cy3 signal, suggesting that the Cy3 dye was physically stacked on the SWCNT surface and therefore photophysically quenched. Upon addition of $100 \mu \mathrm{m}$ dopamine in PBS buffer, the Cy3 signal instantly brightened, and we observed a marked increase in the number of $\mathrm{Cy} 3$ molecules that fluoresced [from $432.9 \pm 11.4$ pre-dopamine to $1601.8 \pm$ 30.9 post-dopamine (mean $\pm \mathrm{SE}$ ); Figure $4 \mathrm{a}$ ]. The time scale for the Cy3 brightening and SWCNT nIR brightening shown in Figure 4 was instantaneous within the $0.5 \mathrm{~s}$ frame rate, suggesting that both processes [dopamine detection via SWCNT brightening and desorption of the Cy3 dye on the $(\mathrm{GT})_{15}$ terminus] occur simultaneously. These results suggest 
that dopamine interacts with the terminal end of the $(\mathrm{GT})_{15}-$ Cy3 DNA in the process of dopamine sensing. To confirm that dequenching of $\mathrm{Cy} 3$ occurs when the fluorophore destacks from the SWCNT surface, we performed a positive control to confirm simultaneous dequenching and destacking for (GT) $)_{15}$ Cy3-SWCNT. To the same surface-immobilized (GT) $)_{15}$ SWCNT we introduced $100 \mathrm{nM}$ unlabeled $(\mathrm{AC})_{6}$ oligonucleotide, which is complementary to the ends of $(\mathrm{GT})_{15}-\mathrm{Cy} 3$ DNA. Upon introduction of the $(\mathrm{AC})_{6}$ oligonucleotide to the microfluidic channel, we observed the same dequenching of the terminal Cy3 dye from the SWCNT surface [from 10.4 \pm 0.8 pre- $(\mathrm{AC})_{6}$ to $60.7 \pm 1.6$ post- $(\mathrm{AC})_{6}($ mean $\pm \mathrm{SE})$; Figure $\left.4 \mathrm{~b}\right]$, albeit at a lower rate due to the slower hybridization dynamics of $(\mathrm{AC})_{6}$ to the terminal end of $(\mathrm{GT})_{15}-\mathrm{Cy} 3$ DNA.

Additional negative controls were performed to ensure that no Cy3 signal was observed in the absence of the Cy3 fluorophore or the SWCNT. First, surface-immobilized $(\mathrm{GT})_{15}$-SWCNT lacking the Cy3 dye upon the introduction of $100 \mu \mathrm{M}$ dopamine showed no signal, as expected (Figure 4c). Second, we confirmed that no change in Cy3 signal was observed when dopamine was introduced to surface-immobilized Cy3- $(\mathrm{GT})_{15}$ DNA without a SWCNT (Figure 4d). Differences in surface coverage due to differences in SWCNT versus DNA surface immobilization were observed (see the Experimental Section), and therefore, our analysis focused on the relative rather than absolute changes in signal. We also established that neither dopamine nor $(\mathrm{AC})_{6}$ alone produced an appreciable signal in the $\mathrm{Cy} 3$ emission channel at its characteristic emission wavelength of $570 \mathrm{~nm}$, and we confirmed that addition of a noncomplementary DNA strand, cDNA (GATGCGTGTCTAAGAT), did not increase the Cy3 fluorescence [from $15.8 \pm 1.5$ pre-cDNA to $13.1 \pm 1.2$ postcDNA (mean $\pm \mathrm{SE})]$.

Reversibility of SWCNT-Based Dopamine Sensors. We tested the reversibility of $(\mathrm{GT})_{15}-\mathrm{SWCNT}$ and $(\mathrm{GU})_{15}$ SWCNT sensors by exposing them periodically to dopamine in a flow chamber. For this purpose, $(\mathrm{GT})_{15}-\mathrm{SWCNT}$ and $(\mathrm{GU})_{15}-$ SWCNT were adsorbed to APTES-functionalized glass slides. The glass slides were mounted into a flow chamber and connected to a pump. The sensors were periodically exposed to $100 \mu \mathrm{M}$ dopamine in PBS and washed with PBS. The results are shown in Figure 5 for $(\mathrm{GT})_{15}-$ SWCNT (also see the movie in the Supporting Information).

We analyzed the fluorescence intensity traces of 2 pixel $\times 2$ pixel $(585 \mathrm{~nm} \times 585 \mathrm{~nm})$ regions, which were most likely single SWCNTs. Representative traces for three sensors are shown in Figure $5 b-d$. Whenever the sensors were exposed to dopamine, their fluorescence intensity increased by a factor of $5.39 \pm 1.44$ (mean $\pm \mathrm{SD})$ for $(\mathrm{GT})_{15}-\mathrm{SWCNT}$ (analysis of first response peak for $n=20$ sensors). The fluorescence of $(\mathrm{GU})_{15}$-SWCNT increased by a factor of $3.67 \pm 1.18$. When the surface was washed with PBS, the fluorescence returned approximately to the starting intensity. However, a slight increase in the base intensity level was observed over the time course of seven dopamine additions. When the washing time was extended to $>30 \mathrm{~min}$, the initial intensity level was recovered. The time constants for recovery of the halfmaximum intensity after washing with PBS were $49 \pm 34 \mathrm{~s}$ for $(\mathrm{GT})_{15}-$ SWCNT and $34 \pm 19 \mathrm{~s}$ for $(\mathrm{GU})_{15}-$ SWCNT. It should be noted that the return to the base level is convoluted by the concentration profile of dopamine that forms in the tubing/flow chamber, so the real recovery times are likely shorter than noted above.
Selectivity of Fluorescent SWCNT-Based Dopamine Sensors. The response of the $(\mathrm{GT})_{15}-\mathrm{SWCNT}$ sensor to molecules with structural or functional homology to dopamine can help reveal the signal transduction mechanism by comparison of the structures and basic chemical properties of various analytes. We studied the influence of structural homologues and substances such as uric acid that are known to interfere with existing electrochemical dopamine sensors. ${ }^{28}$ For this purpose, we first added possible interfering chemicals $(100 \mu \mathrm{M})$ to a $1 \mathrm{mg} / \mathrm{L}$ solution of $(\mathrm{GT})_{15}-\mathrm{SWCNT}$ in PBS (Figure 6a). (-)-Epinephrine and (-)-norepinephrine showed

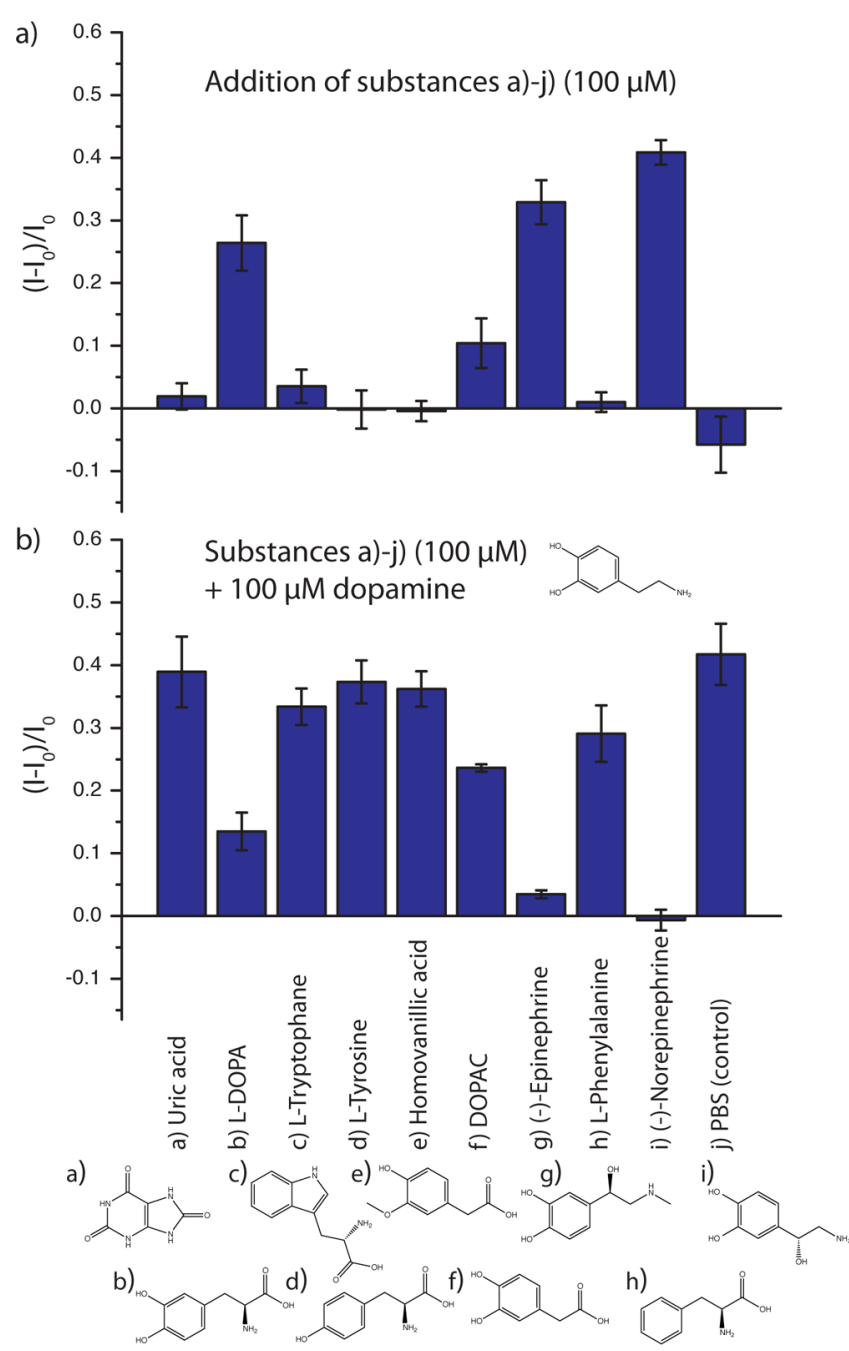

Figure 6. Interference of $(\mathrm{GT})_{15}-\mathrm{SWCNT}$ by compounds of similar structure. (a) Sensor response $\left(I-I_{0}\right) / I_{0}$ after addition of possible interfering molecules $(100 \mu \mathrm{M})$. (b) Sensor response $\left(I-I_{0}\right) / I_{0}$ after addition of $100 \mu \mathrm{M}$ dopamine to solutions already containing the interfering substances. The sensor response is additive with a maximum possible fluorescence increase. Errors are standard deviations.

a similar response as dopamine. L-3,4-Dihydroxyphenylalanine (L-DOPA) produced a medium response, and 3,4-dihydroxyphenylacetic acid (DOPAC) produced a small response relative to dopamine. On the other side, uric acid, L-tryptophan, L-tyrosine, homovanillic acid, and L-phenylalanine showed no significant response.

To the sensors pre-exposed to these dopamine homologues, $100 \mu \mathrm{M}$ dopamine was added to gauge whether a response to 
a)

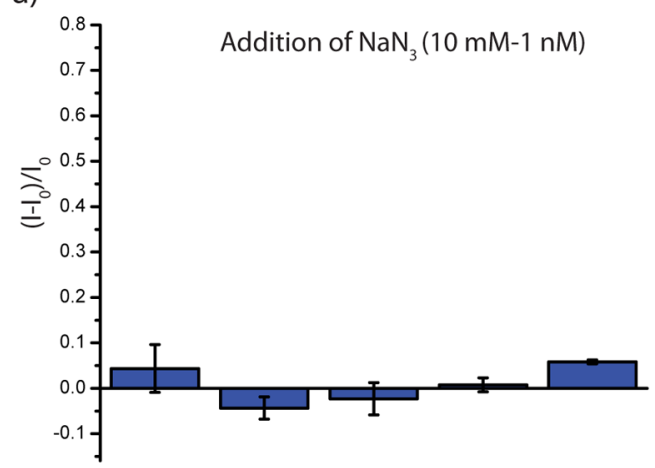

b) ${ }^{0.8} \mathrm{~T} \quad$ Different $\mathrm{NaN}_{3}$ concentrations

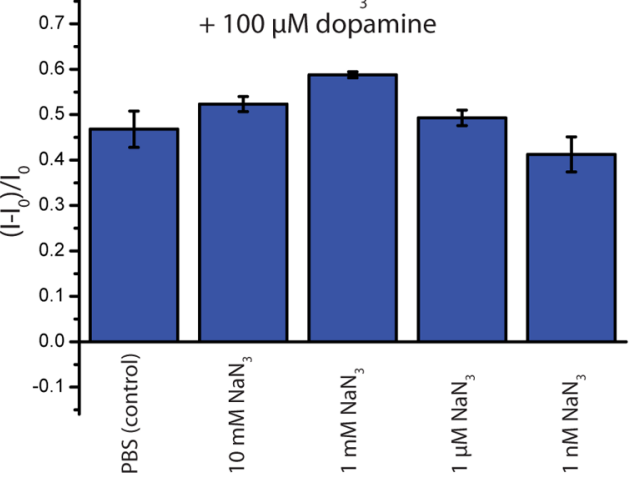

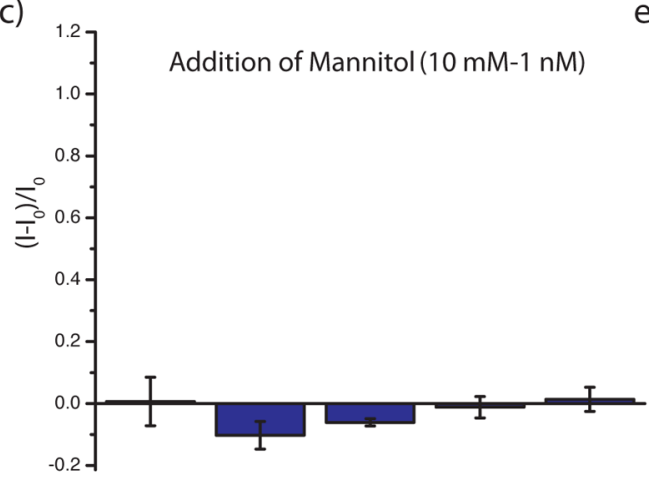

d)

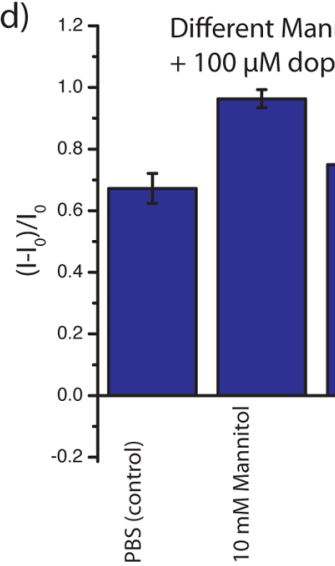

e)

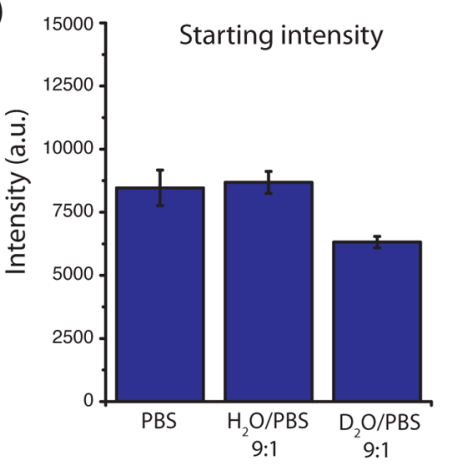

f)

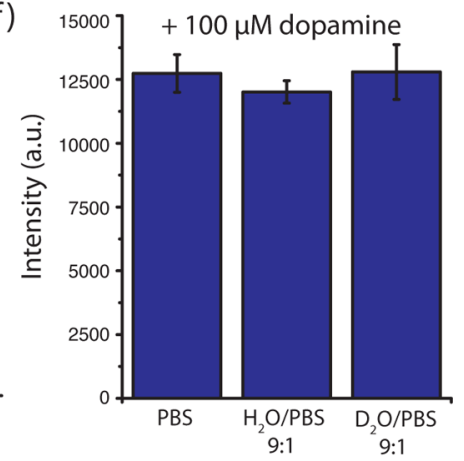

Figure 7. Impact of reactive oxygen species on (GT) $)_{15}-\mathrm{SWCNT}$ dopamine response. (a) Addition of the singlet-oxygen scavenger $\mathrm{NaN}_{3}$ at different concentrations. (b) Consecutive addition of dopamine to these $\mathrm{NaN}_{3}$ containing solutions. (c) Addition of the hydroxyl radical scavenger mannitol at different concentrations. (d) Consecutive addition of dopamine to these mannitol-containing solutions. (e) Fluorescence intensities in buffers containing $90 \% \mathrm{D}_{2} \mathrm{O} . \mathrm{D}_{2} \mathrm{O}$ reduced the fluorescence by more than $25 \%$. (f) Addition of dopamine showed no significant difference between the final intensities in $\mathrm{D}_{2} \mathrm{O}$ - vs $\mathrm{H}_{2} \mathrm{O}$-containing buffers. Errors are standard deviations.

dopamine could occur in their presence (Figure 6b). The results showed that the sensor response is additive with respect to interfering molecules such that if an interfering substance increased the $(\mathrm{GT})_{15}-\mathrm{SWCNT}$ fluorescence, the absolute dopamine response was smaller. This finding can be explained by occupation of dopamine binding sites $\theta$ by interfering substances.

Another possible interferent is ascorbic acid, which is found abundantly in the brain. Ascorbic acid increased the fluorescence of $(\mathrm{GT})_{15}-$ SWCNT but had a $K_{\mathrm{d}}$ of $18.3 \mu \mathrm{M}$, which is 2 orders of magnitude larger than that for dopamine (SI Figure S3).

Role of Reactive Oxygen Species. Dopamine is an aromatic and redox-active molecule. Therefore, a possible explanation for the fluorescence increase of $(\mathrm{GT})_{15}-\mathrm{SWCNT}$ in the presence of dopamine could be dopamine-induced scavenging of reactive oxygen species, as ROS reduce the fluorescence of SWCNTs. ${ }^{29}$ We conducted a series of experiments with ROS scavengers to rule out the influence of ROS. If ROS play a role in SWCNT fluorescence, the presence of a scavenger molecule should act in the place of dopamine as a ROS scavenger, and dopamine would no longer create a sensor response.

First, we added different concentrations of the singlet-oxygen scavenger sodium azide $\left(\mathrm{NaN}_{3}\right)$ or the hydroxyl radical scavenger mannitol to a $1 \mathrm{mg} / \mathrm{L}(\mathrm{GT})_{15}-\mathrm{SWCNT}$ solution in PBS (Figure 7a,c). We observed only small changes in SWCNT fluorescence $(<5 \%)$ for $\mathrm{NaN}_{3}(1 \mathrm{nM})$ and a $<10 \%$ change for mannitol at the highest scavenger concentration (10
$\mathrm{mM})$. To the scavenger $+(\mathrm{GT})_{15}-\mathrm{SWCNT}$ solutions, we added dopamine to a final concentration of $100 \mu \mathrm{M}$ (Figure $7 \mathrm{~b}, \mathrm{~d})$. Although there were small differences in the sensor response, the differences were only subtle or within the error bars. The dopamine sensor response was higher at high mannitol concentrations $(10 \mathrm{mM}, 100$ times more than dopamine) because of the lowering of the starting intensity by mannitol, but at smaller scavenger concentrations, no changes in fluorescence were observed. Experiments with other scavengers such as sorbitol, benzoic acid, and dimethyl sulfoxide showed similar results (SI Figure S5).

We also tested for the presence of singlet oxygen production and interference by repeating the experiments in $\mathrm{D}_{2} \mathrm{O}$. Heavy water increases the lifetime of singlet oxygen by an order of magnitude. $^{30}$ Therefore, if ROS play a role in dopamine detection, a drastic sensor response change for heavy water experiments would be expected. We mixed the $(\mathrm{GT})_{15}-$ SWCNT in a PBS $/ \mathrm{D}_{2} \mathrm{O}(1: 9)$ solution and measured $(\mathrm{GT})_{15}-$ SWCNT fluorescence intensities before (Figure 7e) and after the addition of dopamine (Figure 7f). $\mathrm{D}_{2} \mathrm{O}$ decreased the fluorescence starting intensity but did not have a significant influence on the final intensity after addition of $100 \mu \mathrm{M}$ dopamine.

\section{DISCUSSION}

Detection of neurotransmitters is a challenging analytical problem. To date, only a few methods that can image neurotransmitter release have been reported. ${ }^{31}$ Nevertheless, 
neurotransmitters are a very important class of biomolecules, and nanosensors can facilitate new insights in neurobiology.

We used a new approach for the detection of neurotransmitters that is based on corona phase molecular recognition ( $\mathrm{CoPhMoRe}$ ) of fluorescent single-walled carbon nanotubes (SWCNTs). ${ }^{2}$ For this purpose, SWCNTs were functionalized with different polymers. Through a screening, we investigated how nine different neurotransmitters affect the nIR fluorescence signature of 30 SWCNT-polymer hybrid structures. Interestingly, we found several polymer-SWCNT hybrids whose nIR fluorescence intensities changed in the presence of neurotransmitters (Figure 1a). In theory, every polymer-SWCNT hybrid structure whose nIR fluorescence changes in the presence of a neurotransmitter is a possible sensor, yet a certain degree of specificity for the analyte is also required. We attribute the selectivity of these sensors to the $\mathrm{CoPhMoRe}$ provided by the organic phase around the carbon nanotube. In this scheme, it is the organic corona phase that controls how the analyte influences the fluorescence of the SWCNTs. For example, the presence of a particular analyte may impart conformational changes in the organic corona phase around the SWCNT that lead to quenching or dequenching of the fluorescence, thereby imparting the sensor with selectivity and sensitivity for that particular analyte.

A central finding of our screening approach is that nucleic acid-wrapped carbon nanotubes brighten in the nIR in the presence of catecholamines such as dopamine or (-)-epinephrine. In contrast, most SWCNTs coated with phospholipid derivatives show either no change or a decrease in nIR fluorescence. Therefore, nucleic acid-wrapped SWCNTs have a high potential for use as dopamine sensors with high specificity and sensitivity. It is well-known that dopamine is a reactive molecule and undergoes oxidation to dopamine quinone and other products readily. ${ }^{23,25,26,32} \mathrm{We}$ attribute the time-dependent response (Figure $3 b$ ) to these reactions. Interestingly, polymerization of dopamine might have been a primary contributor to results observed in previous work from our lab, where dopamine was shown to quench the SWCNT fluorescence of DNA-wrapped SWCNTs. ${ }^{2,4}$ These experiments were performed at higher dopamine concentrations $(500 \mu \mathrm{M})$ and after longer incubation times, conditions under which dopamine is known to polymerize readily. It is likely that in previous publications, a complex mixture of polymerized dopamine instead of pure dopamine was measured and that SWCNTs aggregated/precipitated and reduced the fluorescence count. When our samples were measured several hours or 1 day after the addition of dopamine, we also observed a strong decrease in fluorescence. In contrast, herein we show results based on more biologically pertinent dopamine concentrations and at time scales that are more relevant for neuronal studies: the present experiments were conducted at much lower dopamine concentrations $(<100 \mu \mathrm{M})$ with fresh dopamine solutions at shorter incubation times (seconds or minutes) and revealed that dopamine and catecholamines in general increase the fluorescence of nucleic acid-wrapped SWCNTs, with the magnitude of the fluorescence increase depending on the nucleic acid sequence (Figure 1a). The calibration curve of the $(\mathrm{GT})_{15}-\mathrm{SWCNT}$ dopamine sensor (Figure 3a) shows that the lower bound of dopamine concentrations that can be detected is in the nanomolar range. While we always prepared fresh dopamine solutions for each experiment, the slight variations in sensor responses are likely due to small variations in the time to prepare the solution, prepare the wells, and measure the sample.

The polymer-SWCNT hybrids with the highest relative fluorescence increases were those with the DNA sequence $(\mathrm{GT})_{15}$ and the RNA sequence $(\mathrm{GU})_{15}$. In solution, $100 \mu \mathrm{M}$ dopamine produced an up to $80 \%$ increase in the fluorescence. This increase in fluorescence intensity is the basis for a turn-on dopamine sensor. In contrast, previously reported SWCNTbased sensors for small molecules are based on SWCNT fluorescence quenching. ${ }^{4,6}$

A feasible mechanism for dopamine sensing could potentially involve covalent modification of the SWCNT. First and foremost, we ruled out this possibility by verifying that the Raman spectra of the SWCNTs before and after the addition of dopamine remained invariant, as did the D/G Raman peak ratio (SI Figure S1). Thus, a mechanism based on covalent modification of the SWCNT can be ruled out. In addition, the reversibility of the sensing response indicates that neither the SWCNT nor the nucleic acid wrapping are covalently modified by dopamine (Figure 5). We have also verified that the SWCNT absorption cross section is invariant with dopamine addition, confirming that the quantum yield of (GT) ${ }_{15}$-SWCNT increases in the presence of dopamine (SI Figures S1 and S2). Additionally, scavenging of reactive oxygen species by dopamine was ruled out as the main mechanism by experiments with competing reactive oxygen scavengers (Figure 7 and SI Figure S5).

Probing the local structure of the $(\mathrm{GT})_{15}$ polymer via fluorophore tagging of the DNA polymer $5^{\prime}$ end strongly suggested that a small local perturbation of the polymer ends occurs when dopamine enters the organic corona of the SWCNT (Figure 4). In this manner, the interaction of dopamine with the nucleic acid or the SWCNT surface perturbs the nucleic acid conformation. This conformational change decreases SWCNT exciton decay routes, leading to an increase in SWCNT fluorescence (quantum yield). It is likely that the nucleic acid conformational changes lead to removal of quenching species from the solvent or quenching groups of the nucleic acid backbone from the surface of the SWCNT. For instance, protons are known to quench SWCNT fluorescence, and their removal could explain the increase in fluorescence. ${ }^{33}$

In this picture, the selectivity of the sensor response is determined by the local polymer structure within the SWCNT corona phase. Our results show that even small differences in the nucleic acid sequence cause strong changes in the sensor response (Figure 1a) and that dopamine homologues exhibit different responses compared with dopamine. For instance, both (GT) $)_{15}-$ SWCNT and its RNA analogue $(\mathrm{GU})_{15}$ SWCNT show a strong dopamine response (79\% vs $44 \%$ fluorescence increase) but a completely different response to the catecholamine (-)-epinephrine ( $62 \%$ vs $\sim 0 \%$ fluorescence increase) (Figure 1a). All these data support the CoPhMoRe concept and suggest that further engineering and screening of the organic phase of the SWCNT will provide even more selective and sensitive sensors.

The interaction between the nucleic acid-SWCNT and dopamine could involve either direct physical contact or a redox reaction. A redox reaction of the nucleic acid polymer is likely to change the polymer structure within the SWCNT corona phase, thus changing the quantum yield of SWCNT fluorescence. To understand the details of this mechanism, it is noteworthy that dopamine is easily oxidized to dopamine quinone, which can undergo further downstream reactions. ${ }^{26}$ 
Additional evidence for redox-chemistry-based CoPhMoRe is provided by the redox potentials of compounds similar to the dopamine analyte that produce similar SWCNT fluorescence responses (SI Figure S6). For instance, ascorbic acid has a similar redox potential but a different structure compared with dopamine and also produces a response (SI Figure S4). L-Dopa and DOPAC produce sensor responses similar to that of dopamine but have similar structures and redox potentials. In contrast, uric acid has a different structure but a redox potential that is only slightly larger than that of dopamine, but it produces no response when added to $(\mathrm{GT})_{15}-$ SWCNT. Plotting sensor responses against the redox peak current potentials (from cyclic voltammetry data) shows that the redox potential of the analyte can play a role in SWCNT-based sensing (SI Figure S6).

This hypothesis is also in agreement with our finding that $(\mathrm{GT})_{15}-$ SWCNT showed a higher response than $(\mathrm{AT})_{15}$ SWCNT $\left[0.79\right.$ for $(\mathrm{GT})_{15}$ vs $0.15(\mathrm{AT})_{15}$; Figure 1a], since the guanine nucleotide is known to act as a oxidation sink in nucleic acids, and therefore, higher guanine content should lead to higher sensor responses. ${ }^{34}$

Recently it was reported that DNA-functionalized SWCNTs show a fluorescence increase in the presence of reducing agents such as trolox or mercaptoethanol. ${ }^{35}$ These findings along with ours indicate that substances that are readily oxidized can generally increase the fluorescence of polynucleotide-functionalized SWCNTs, although exact mechanisms might be different for different substances.

A redox-based mechanism presupposes that the nucleic acid is already oxidized before dopamine is added and is then reduced by dopamine. However, the reversibility of the sensor response (Figure 5) implies that there is an oxidized equilibrium state of $(\mathrm{GT})_{15}$ DNA on the SWCNT and that the nucleotide radical cations do not irreversibly react in this state, which is rather unlikely. Dopamine also shares an aromatic structure with L-Dopa, DOPAC, (-)-epinephrine, and (-)-norepinephrine. Therefore, another plausible mechanism of the molecular recognition could be based on $\pi-\pi$ stacking of the aromatic ring of dopamine with the SWCNT. In this picture, the DNA sequence serves as a diffusion barrier, thus providing selectivity. When dopamine binds to the SWCNT surface it displaces parts of the DNA backbone, which increases the fluorescence quantum yield.

Although the exact nature of the dopamine-sensor interaction remains under investigation, both mechanisms are compatible with the CoPhMoRe concept because it does not presuppose the type of the interaction between the organic corona and the analyte.

In this work we focused on the development and characterization of a dopamine sensor, but the screening in Figure 1a clearly shows many other promising polymerSWCNT sensors for other neurotransmitters. Future work will focus on increasing the sensitivity and selectivity of the sensors for dopamine and other neurotransmitters using various parallel approaches. First, we envision the development of ratiometric sensors using different SWCNT chiralities to expand the library of neurotransmitters that we can detect. In this work we used HiPCO SWCNTs, which comprise a mixture of all SWCNT chiralities, not all of which can be excited simultaneously. The use of single-chirality samples should increase the sensitivity of future dopamine sensors by enabling excitation of all SWCNTs within a sample. Combinations of different SWCNT chiralities could also be used to synthesize ratiometric sensors by using a combination of two or more different polymer-SWCNT samples to gain higher selectivity and sensitivity through simultaneous multiplexed detection.

Tools in neuroscience for the optical detection of dopamine are lacking, particularly at the nanoscale. For instance, there are no tools currently available for direct measurements of dopamine concentrations in the synaptic cleft. Fast-scan cyclic voltammetry measurements in combination with geometrical assumptions suggest that extracellular dopamine levels are 250 $\mathrm{nM}$ in the nucleus accumbens of the rat, $1.6 \mathrm{mM}$ (transient) in the synaptic cleft, and $25 \mathrm{mM}$ in dopamine-containing vesicles. $^{36}$ Our optical dopamine sensor could be used to measure static dopamine concentrations and also dynamic changes in dopamine concentrations with high spatial and temporal precision. Additionally, our sensor could be used to track the secretion of dopamine from dopaminergic neurons, providing a fundamental understanding of how neuronal cells communicate with each other.

In addition to its use as a sensor, the brightening of nucleic acid-wrapped SWCNTs in the presence of dopamine/catecholamines can be helpful for SWCNT imaging in general. Figure 5 shows that single $(\mathrm{GT})_{15}-$ SWCNTs get brighter by a factor of $>5$. Therefore, adding fresh dopamine to nucleic acid-wrapped SWCNTs, particularly $(\mathrm{GT})_{15}-$ SWCNTs, might be useful to achieve higher fluorescence signals for applications requiring bright SWCNTs, such as single particle imaging.

Lastly, it is important to note that this work presents a novel approach to SWCNT-based sensor development. While previous SWCNT sensor developments have relied on known biological recognition elements such as antibodies, the work presented here is based on CoPhMoRe provided by polymers that have no pre-existing affinity for dopamine. We envision that future generations of SWCNT-based sensors will not be limited to using pre-existing molecular recognition elements and instead will exploit molecular recognition provided by the structure of the corona phase of a SWCNT-polymer conjugate. Through further screening rounds of additional polymer-SWCNT hybrids, the selectivity and specificity of these sensors can be further improved, and novel sensors can be discovered for which there currently exist no naturally occurring biological recognition elements.

\section{CONCLUSION}

This work presents a new method for the optical detection of neurotransmitters by using fluorescent single-walled carbon nanotubes (SWCNTs). Our approach is based on chemical engineering of the organic phase (corona) around the carbon nanotube, resulting in corona phase molecular recognition (CoPhMoRe) of neurotransmitter analytes. By using different polymers wrapped around the carbon nanotube, we can generate polymer-SWCNT hybrids that are able to detect various neurotransmitters. One specific finding of our screening shows that $(\mathrm{GT})_{15}$ DNA- and (GU) ${ }_{15}$ RNA-wrapped SWCNTs can serve as highly selective and sensitive fluorescence turn-on sensors for catecholamine neurotransmitters such as dopamine. Our approach incorporates three very important figures of merit for sensor development: the sensors provide a strong optical turn-on response in the nIR, are highly sensitive and reversible, and are fully functional at the nanoscale. Mechanistic studies have shown that the nucleic acid backbone most likely acts as a reversible dopamine-dependent switch that can modulate exciton decay routes and thereby the SWCNT fluorescence. In the future, these sensors could be used for 
spatiotemporal neurotransmitter detection near synapses or in neural networks.

\section{ASSOCIATED CONTENT}

\section{S Supporting Information}

Raman spectra, absorption spectra, and absorption kinetics (Figures S1 and S2); schematic of the optical setup (Figure S3); ascorbic acid calibration curves (Figure S4); additional ROS scavenger experiments (Figure S5); and plot of sensor response versus redox potential (Figure S6). This material is available free of charge via the Internet at http://pubs.acs.org.

\section{AUTHOR INFORMATION}

\section{Corresponding Author}

strano@mit.edu

\section{Author Contributions}

${ }^{\dagger}$ S.K. and M.P.L. contributed equally.

\section{Notes}

The authors declare no competing financial interest.

\section{ACKNOWLEDGMENTS}

This work was supported primarily by the National Science Foundation under Award 1213622. S.K. was supported by a postdoctoral fellowship from the Deutsche Forschungsgemeinschaft (DFG). M.P.L. acknowledges an NSF postdoctoral research fellowship under Award 1306229.

\section{REFERENCES}

(1) Davie, C. A. Br. Med. Bull. 2008, 86, 109.

(2) Zhang, J.; Landry, M. P.; Barone, P. W.; Kim, J. H.; Lin, S.; Ulissi, Z. W.; Lin, D.; Mu, B.; Boghossian, A. A.; Hilmer, A. J.; Rwei, A.; Hinckley, A. C.; Kruss, S.; Shandell, M. A.; Nair, N.; Blake, S.; Sen, F.; Sen, S.; Croy, R. G.; Li, D.; Yum, K.; Ahn, J. H.; Jin, H.; Heller, D. A.; Essigmann, J. M.; Blankschtein, D.; Strano, M. S. Nat. Nanotechnol. 2013, 8, 959.

(3) Kruss, S.; Hilmer, A. J.; Zhang, J.; Reuel, N. F.; Mu, B.; Strano, M. S. Adv. Drug Delivery Rev. 2013, 65, 1933.

(4) Zhang, J.; Boghossian, A. A.; Barone, P. W.; Rwei, A.; Kim, J. H.; Lin, D.; Heller, D. A.; Hilmer, A. J.; Nair, N.; Reuel, N. F.; Strano, M. S. J. Am. Chem. Soc. 2011, 133, 567.

(5) O'Connell, M. J.; Bachilo, S. M.; Huffman, C. B.; Moore, V. C.; Strano, M. S.; Haroz, E. H.; Rialon, K. L.; Boul, P. J.; Noon, W. H.; Kittrell, C.; Ma, J.; Hauge, R. H.; Weisman, R. B.; Smalley, R. E. Science 2002, 297, 593.

(6) Jin, H.; Heller, D. A.; Kalbacova, M.; Kim, J. H.; Zhang, J.; Boghossian, A. A.; Maheshri, N.; Strano, M. S. Nat. Nanotechnol. 2010, 5, 302 .

(7) Barone, P. W.; Parker, R. S.; Strano, M. S. Anal. Chem. 2005, 77, 7556.

(8) Heller, D. A.; Baik, S.; Eurell, T. E.; Strano, M. S. Adv. Mater. 2005, 17, 2793.

(9) Heller, D. A.; Jin, H.; Martinez, B. M.; Patel, D.; Miller, B. M.; Yeung, T. K.; Jena, P. V.; Hobartner, C.; Ha, T.; Silverman, S. K.; Strano, M. S. Nat. Nanotechnol. 2009, 4, 114.

(10) Adams, K. L.; Puchades, M.; Ewing, A. G. Annu. Rev. Anal. Chem. 2008, 1, 329.

(11) Kim, D.; Koseoglu, S.; Manning, B. M.; Meyer, A. F.; Haynes, C. L. Anal. Chem. 2011, 83, 7242.

(12) Savtchenko, L. P.; Rusakov, D. A. Proc. Natl. Acad. Sci. U.S.A. 2007, 104, 1823.

(13) Wightman, R. M.; Jankowski, J. A.; Kennedy, R. T.; Kawagoe, K. T.; Schroeder, T. J.; Leszczyszyn, D. J.; Near, J. A.; Diliberto, E. J., Jr.; Viveros, O. H. Proc. Natl. Acad. Sci. U.S.A. 1991, 88, 10754.
(14) Rodriguez, P. C.; Pereira, D. B.; Borgkvist, A.; Wong, M. Y.; Barnard, C.; Sonders, M. S.; Zhang, H.; Sames, D.; Sulzer, D. Proc. Natl. Acad. Sci. U.S.A. 2013, 110, 870.

(15) Gubernator, N. G.; Zhang, H.; Staal, R. G.; Mosharov, E. V.; Pereira, D. B.; Yue, M.; Balsanek, V.; Vadola, P. A.; Mukherjee, B.; Edwards, R. H.; Sulzer, D.; Sames, D. Science 2009, 324, 1441.

(16) Masharina, A.; Reymond, L.; Maurel, D.; Umezawa, K.; Johnsson, K. J. Am. Chem. Soc. 2012, 134, 19026.

(17) Okubo, Y.; Sekiya, H.; Namiki, S.; Sakamoto, H.; Iinuma, S.; Yamasaki, M.; Watanabe, M.; Hirose, K.; Iino, M. Proc. Natl. Acad. Sci. U.S.A. 2010, 107, 6526.

(18) Marvin, J. S.; Borghuis, B. G.; Tian, L.; Cichon, J.; Harnett, M. T.; Akerboom, J.; Gordus, A.; Renninger, S. L.; Chen, T. W.; Bargmann, C. I.; Orger, M. B.; Schreiter, E. R.; Demb, J. B.; Gan, W. B.; Hires, S. A.; Looger, L. L. Nat. Methods 2013, 10, 162.

(19) Mu, B.; McNicholas, T. P.; Zhang, J.; Hilmer, A. J.; Jin, Z.; Reuel, N. F.; Kim, J. H.; Yum, K.; Strano, M. S. J. Am. Chem. Soc. 2012, $134,17620$.

(20) Lamichhane, R.; Solem, A.; Black, W.; Rueda, D. Methods 2010, 52, 192.

(21) Roy, R.; Hohng, S.; Ha, T. Nat. Methods 2008, 5, 507.

(22) Steinberg, E. E.; Keiflin, R.; Boivin, J. R.; Witten, I. B.; Deisseroth, K.; Janak, P. H. Nat. Neurosci. 2013, 16, 966.

(23) Lee, H.; Dellatore, S. M.; Miller, W. M.; Messersmith, P. B. Science 2007, 318, 426.

(24) Dreyer, D. R.; Miller, D. J.; Freeman, B. D.; Paul, D. R.; Bielawski, C. W. Langmuir 2012, 28, 6428.

(25) Liebscher, J.; Mrówczyński, R.; Scheidt, H. A.; Filip, C.; Hădade, N. D.; Turcu, R.; Bende, A.; Beck, S. Langmuir 2013, 29, 10539.

(26) Lynge, M. E.; van der Westen, R.; Postma, A.; Stadler, B. Nanoscale 2011, 3, 4916.

(27) Yang, R.; Jin, J.; Chen, Y.; Shao, N.; Kang, H.; Xiao, Z.; Tang, Z.; Wu, Y.; Zhu, Z.; Tan, W. J. Am. Chem. Soc. 2008, 130, 8351.

(28) Wightman, R. M.; May, L. J.; Michael, A. C. Anal. Chem. 1988, $60,769 \mathrm{~A}$.

(29) Sen, F.; Boghossian, A. A.; Sen, S.; Ulissi, Z. W.; Zhang, J.; Strano, M. S. ACS Nano 2012, 6, 10632.

(30) Merkel, P. B.; Kearns, D. R.; Nilsson, R. J. Am. Chem. Soc. 1972, 94, 1030.

(31) Sames, D.; Dunn, M.; Karpowicz, R. J.; Sulzer, D. ACS Chem. Neurosci. 2013, 4, 648.

(32) Herlinger, E.; Jameson, R. F.; Linert, W. J. Chem. Soc., Perkin Trans. 2 1995, 259.

(33) Strano, M. S.; Huffman, C. B.; Moore, V. C.; O’Connell, M. J.; Haroz, E. H.; Hubbard, J.; Miller, M.; Rialon, K.; Kittrell, C.; Ramesh, S.; Hauge, R. H.; Smalley, R. E. J. Phys. Chem. B 2003, 107, 6979.

(34) Steenken, S.; Jovanovic, S. V. J. Am. Chem. Soc. 1997, 119, 617.

(35) Lee, A. J.; Wang, X.; Carlson, L. J.; Smyder, J. A.; Loesch, B.; Tu, X.; Zheng, M.; Krauss, T. D. Nano Lett 2011, 11, 1636.

(36) Garris, P. A.; Ciolkowski, E. L.; Pastore, P.; Wightman, R. M. J. Neurosci. 1994, 14, 6084. 